\title{
Astrocytes from the Contused Spinal Cord Inhibit Oligodendrocyte Differentiation of Adult Oligodendrocyte Precursor Cells by Increasing the Expression of Bone Morphogenetic Proteins
}

\author{
Yaping Wang, ${ }^{2,3,5 *}$ Xiaoxin Cheng, ${ }^{1,2,3 *}$ Qian He, ${ }^{1,2,3}$ Yiyan Zheng, ${ }^{1}$ Dong H. Kim, ${ }^{1}$ Scott R. Whittemore, ${ }^{2,3,4}$ \\ and Qilin L. Cao ${ }^{1,2,3}$ \\ ${ }^{1}$ The Vivian L Smith Department of Neurosurgery, University of Texas Medical School at Houston, Houston, Texas 77030; ${ }^{2}$ Kentucky Spinal Cord Injury \\ Research Center, and Departments of ${ }^{3}$ Neurological Surgery and ${ }^{4}$ Anatomical Sciences and Neurobiology, University of Louisville School of Medicine, \\ Louisville, Kentucky 40202, and 5Department of Anesthesiology, the Second Xianya Hospital, Central South University Xianya Medical School, Changsha, \\ Hunan 410011, Peoples Republic of China
}

Promotion of remyelination is an important therapeutic strategy to facilitate functional recovery after traumatic spinal cord injury (SCI). Transplantation of neural stem cells (NSCs) or oligodendrocyte precursor cells (OPCs) has been used to enhance remyelination after SCI. However, the microenvironment in the injured spinal cord is inhibitory for oligodendrocyte (OL) differentiation of NSCs or OPCs. Identifying the signaling pathways that inhibit OL differentiation in the injured spinal cord could lead to new therapeutic strategies to enhance remyelination and functional recovery after SCI. In the present study, we show that reactive astrocytes from the injured rat spinal cord or their conditioned media inhibit OL differentiation of adult OPCs with concurrent promotion of astrocyte differentiation. The expression of bone morphogenetic proteins (BMP) is dramatically increased in the reactive astrocytes and their conditioned media. Importantly, blocking BMP activity by BMP receptor antagonist, noggin, reverse the effects of active astrocytes on OPC differentiation by increasing the differentiation of OL from OPCs while decreasing the generation of astrocytes. These data indicate that the upregulated bone morphogenetic proteins in the reactive astrocytes are major factors to inhibit OL differentiation of OPCs and to promote its astrocyte differentiation. These data suggest that manipulation of BMP signaling in the endogenous or grafted NSCs or OPCs may be a useful therapeutic strategy to increase their OL differentiation and remyelination and enhance functional recovery after SCI.

\section{Introduction}

Demyelination is one of major contributors to pathophysiology of many neurological diseases, including multiple sclerosis (MS) and spinal cord injury (SCI) (Franklin and ffrench-Constant, 2008). Although remyelination is observed after the acute demyelination lesion in the early stage of multiple sclerosis, it becomes incomplete and eventually fails even though oligodendrocyte precursor cells (OPCs) are present in the demyelinated areas (Trapp and Nave, 2008). OPCs retain their capacity to differentiate into mature oligodendrocytes to remyelinate demyelinated axons, but do not do so. Similarly, OPCs also fail to mature into myelinating oligodendrocytes after transplantation into the chronically injured spinal cord, even though the demyelinated

\footnotetext{
Received Nov. 6, 2009; revised Dec. 7, 2010; accepted Dec. 11, 2010.

This research was supported by National Institutes of Health Grants NS061975 (Q.L.C.), NS054708 (S.R.W.), and RR15576 (Q.L.C., SRW), The TIRR Foundation through Mission Connect (Q.L.C.), the Kentucky Spinal Cord and Head Injury Research Trust (Q.L.C., S.R.W.), and the Commonwealth of Kentucky Research Challenge for Excellence Trust Fund (S.R.W.).

*Y.W. and X.C. contributed equally.

Correspondence should be addressed to Dr. Qilin Cao, Department of Neurosurgery, University of Texas Medical School at Houston, 6341 Fannin Street, MSE R158, Houston, TX 77030. E-mail:qi-lin.cao@uth.tmc.edu. DOI:10.1523/JNEUROSCI.5524-09.2011

Copyright $@ 2011$ the authors $\quad 0270-6474 / 11 / 316053-06 \$ 15.00 / 0$
}

axons are available for remyelination (Keirstead et al., 2005). Understanding why remyelination fails within demyelinated lesions could lead to therapeutic targets for many neurological diseases involved in demyelination.

After demyelinating lesions, as well as other neurological diseases, one dramatic physiopathological change is gliosis, which may play important roles in remyelination. In chronic MS plaques, an absence of remyelination is accompanying by robust astrogliosis. This is in contrast to acute MS lesions in which an absence of sclerosis is correlated with widespread remyelination (Raine, 2008). The correlation between astrogliosis and persistent demyelination has also been found in cuprizone-induced experimental demyelination (Skripuletz et al., 2010), experimental allergic encephalomyelitis (Anderson et al., 2008), and traumatic SCI (Keirstead et al., 2005). These studies suggest that astrogliosis may contribute to the failure of remyelination. In this study, we purified astrocytes from the normal adult spinal cord, acute and chronic injured spinal cord and directly tested their effects on oligodendrocyte (OL) differentiation of OPCs. Our results showed that astrocytes from the injured spinal cord inhibited OL maturation of OPCs and bone morphogenetic protein (BMP) signaling is one of the major mediators of this inhibition of OL maturation. 


\section{Materials and Methods}

Isolation of OPCs from adult spinal cord. OPCs were immunopanned with an $\mathrm{O} 4$ antibody from adult spinal cord of Fischer rats expressing human placental alkaline phosphatase (hPAP) as described previously (Kisseberth et al., 1999). Briefly, the dissected spinal cords were minced into 1 $\mathrm{mm}^{3}$ pieces and incubated in HBSS containing $0.1 \%$ papain, $0.1 \%$ neutral protease, and $0.01 \%$ DNase for $30 \mathrm{~min}$ at $37^{\circ} \mathrm{C}$. The digestion was stopped by addition of an equal volume of DMEM containing $20 \%$ fetal bovine serum. Tissues were dissociated by repeated trituration with firepolished Pasteur pipettes and were filtered through $70 \mu \mathrm{m}$ nylon mesh. The cells were incubated on an anti-RAN-2 antibody-coated dish for 30 min to deplete type- 1 astrocytes and meningeal cells and then transferred to an O4-coated dish for $45 \mathrm{~min}$ to select OPC cells. The purified OPCs were cultured in poly-L-lysine/laminin (P/L)-coated dishes with DMEM/ F12 medium containing $1 \times \mathrm{N} 2$ and $1 \times$ B27 supplements, FGF2 (20 $\mathrm{ng} / \mathrm{ml})$, PDGF-aa $(10 \mathrm{ng} / \mathrm{ml})$, insulin $(5 \mu \mathrm{g} / \mathrm{ml})$ and BSA (0.1\%). Cells were fed with fresh growth medium every other day. In all cases, an aliquot of cells was analyzed the next day to determine the efficiency of the immunopanning. Only those cell preparations in which $>95 \%$ of the bound cells expressed $\mathrm{O} 4$ were used in the experiments.

Purification of astrocytes. Adult female Fischer 344 rats were anesthetized with Nembutal (50 mg/kg, i.p.) and received a dorsal laminectomy at the ninth thoracic vertebral level (T9) to expose the spinal cord, and then a 150 kdyn contusive SCI using the Infinite Horizons impactor (Precision Systems and Instrumentation). At $7 \mathrm{~d}$ or 1 month after injury, a two $\mathrm{cm}$ spinal cord segment spanning the injury epicenter or the whole thoracic spinal cord from normal rats were dissected and diced into 1 $\mathrm{mm}^{3}$ pieces in Hanks' balanced salt solution (HBSS) and 10 mM HEPES solution, followed by enzymatic digestion in HBSS/HEPES containing $0.01 \%$ papain, $0.1 \%$ trypsin and $0.01 \%$ DNase I for $30 \mathrm{~min}$ at $37^{\circ} \mathrm{C}$ with occasional shaking. Enzymatic digestion was stopped by the addition of an equal volume of DMEM containing 20\% fetal bovine serum. Tissues were dissociated by repeated trituration with fire-polished Pasteur pipettes and were filtered through $70 \mu \mathrm{m}$ nylon mesh, centrifuged, and resuspended in astrocyte medium (DMEM $+10 \%$ FBS $+1 \times \mathrm{N} 2)$. The isolated cells were plated in T75 flasks and cultured in a $37^{\circ} \mathrm{C}, 5 \% \mathrm{CO}_{2}$ incubator until cells reached confluence after 10-14 d. The flasks were then shaken at 275 rotations $/ \mathrm{min}$ overnight at $37^{\circ} \mathrm{C}$ to get rid of microglia, OPC, OLs and neurons. The astrocytes were cultured until reaching confluence and then treated with cytosine arabinoside $(20 \mathrm{~mm})$ for $72 \mathrm{~h}$ to eliminate proliferating cells. To collect conditioned medium (CM), the confluent passage 0 or 1 astrocytes were washed once with DMEM/ F12 and then incubated in DMEM/F12 supplemented with N2, B27 for $48 \mathrm{~h}$. CM was centrifuged, filtered, and concentrated 30 -fold with a Centriplus 30 filter (Amicon), and stored at $-20^{\circ} \mathrm{C}$ until use. The concentrated $\mathrm{CM}$ was diluted to 1:30 in fresh serum-free OPC differentiating medium when use.

Oligodendrocyte differentiation of adult OPCs. Adult OPCs were plated on P/L-coated $60 \mathrm{~mm}$ culture dishes for Western blot analysis or 24 wells plates for immunohistochemistry. The following day, adult OPCs were differentiated in control differentiation medium (DMEM/F12 supplemented with N2, B27), or CMs of astrocytes from normal, 1 week or 1 month injured spinal cord for $3 \mathrm{~d}$. The percentages of $\mathrm{O}^{+}{ }^{+} \mathrm{OLs}$ or glial fibrillary acid protein $(\mathrm{GFAP})^{+}$astrocytes were assessed by immunohistochemistry. For cocultures, purified astrocytes from normal, or 1 week or 1 month injured spinal cord were grown in 24 wells plates and then OPCs were put on the top. After $1 \mathrm{~d}$ in proliferation medium, the cocultures were switched to differentiation medium for three more days. Then the percentages of $\mathrm{O}^{+} \mathrm{OLs}^{+}$or $\mathrm{GFAP}^{+}$astrocytes to the total number of hPAP-OPCs were quantified using immunohistochemistry.

Immunofluorescence in vitro. To detect the surface membrane antigens, cells cultured on 24-well plates were incubated primary antibodies A2B5, O4, O1 (hybridoma supernatant, undiluted; ATCC) or hPAP (AbD Serotec) at $4^{\circ} \mathrm{C}$ for $45 \mathrm{~min}$. After fixation with $4 \%$ paraformaldehyde, cells were incubated in FITC- or Texas Red-conjugated donkey anti-mouse IgM for $1 \mathrm{~h}$ at room temperature. For recognition of other antigens, cells cultured on 24 well plates were fixed with $4 \%$ paraformaldehyde. Mouse monoclonal antibodies against myelin basic protein
(MBP) 1:50 (Millipore) or rabbit polyclonal antibodies against GFAP 1:400 (Sigma), were applied overnight at $4^{\circ} \mathrm{C}$. Then the appropriate fluorophore-conjugated secondary antibodies 1:200 (Jackson ImmunoResearch) were applied and the nuclei were counterstained with $4^{\prime}, 6^{\prime}$ diamidino-2-phenylindole dihydrochloride. Controls were performed with species-specific IgG or sera and with inappropriate secondary antibodies. Both showed negligible background. Total cellular counts for each experimental well were obtained in 10 fields under $20 \times$ objective from three independent culture wells. The result for each experimental condition was verified a minimum of three times.

Western blot experiments. Cells were harvested in ice-cold lysis buffer (containing $25 \mathrm{~mm}$ Tris, pH 7.6, $1 \mathrm{~mm} \mathrm{MgCl}_{2}, 1 \mathrm{~mm}$ EGTA, 1\% Triton $\mathrm{X}-100,1 \% \mathrm{SDS}, 1 \mathrm{~mm}$ PMSF, $50 \mu \mathrm{g} / \mathrm{ml}$ antipain, $2 \mu \mathrm{g} / \mathrm{ml}$ aprotinin, 1 $\mu \mathrm{g} / \mathrm{ml}$ leupeptin, $1 \mu \mathrm{g} / \mathrm{ml}$ pepstatin A). Protein concentration was determined by BCA kit (Pierce Chemical). Equivalent amounts of total protein extract from each sample were mixed with sample buffer, boiled, and loaded onto SDS polyacrylamide gels. Electrophoretic separation of the extracts was typically performed on $7.5-15 \%$ gels (depending on the molecular weight of the protein of interest). The proteins were then transferred to pure nitrocellulose membranes and probed with monoclonal-specific antibodies GFAP (Sigma), MBP (Millipore), PLP (Sigma), Olig1 (Millipore), BMP4 (R\&D Systems) with polyclonal antibodies Olig2, and phosphorylated SMAD 1/5/8 (pSMAD 1/5/8) (Cell Signaling Technology), BMP2 (AbD Serotec). Additionally, an antibody against $\beta$-actin (Sigma) was used as a loading control. Appropriate secondary HRP-conjugated antibodies were used for detection with chemiluminescence ECL reagents (GE Healthcare).

\section{Results}

We used sequential immnunopanning to obtain highly purified OPCs from adult rat spinal cord as described previously (Talbott et al., 2006; Cheng et al., 2007). All cells expressed hPAP as well as OPC markers O4 (Fig. 1A,B), A2B5 (Fig. 1C), Olig2 (Fig. 1B), and Sox 10 (Fig. 1C). Less than $10 \%$ of adult OPCs were positive for the OL-specific proteins O1 or GalC (data not shown). They lacked the staining for astrocyte-specific intermediate filament GFAP (data not shown). After withdrawal of FGF2 and PDGF-aa for $3 \mathrm{~d}$, OPCs differentiated into mature OLs expressing O1 (Fig. $1 D)$. After coculture with DRG neurons for 2 weeks, OPCs matured into MBP-expressing mature OLs to form myelin around axons (Fig. 1E). These results demonstrated that OPCs were functional to differentiate into mature OLs to myelinate axons. Astrocyte differentiation from OPCs was observed only when serum or BMP was added to the differentiation medium.

To test the effects of astrocytes on the differentiation of OPCs, we purified astrocytes from normal spinal cord (NSC-A), 1 week injured spinal cord (1wISC-A), or 1 month injured spinal cord (1mISC-A). After grown in astrocyte medium for 10-14 d before shaking procedure, majority of cells were $\mathrm{GFAP}^{+}$astrocytes with a few $\mathrm{O} 1{ }^{+} \mathrm{OLs}$ and $\mathrm{OX} 42{ }^{+}$microglia (supplemental Fig. 1, available at www.jneurosci.org as supplemental material). Few A2B5 ${ }^{+}$OPCs, but not neurons, were also observed. However, after shaking procedure and Cytosine arabinoside treatment, 99\% of cells were GFAP ${ }^{+}$ or Vimentin-positive astrocytes (supplemental Fig. 1, available at www.jneurosci.org as supplemental material; Fig. $1 F$ ). Therefore, astrocytes can be successfully purified from NSC, 1wISC or 1mISC. The morphology of astrocytes from the normal or injured spinal cord was not very different with majority being protoplasmic and some fibrous. All purified astrocytes were expressing GFAP (Fig. $1 F)$. Over $50 \%$ astrocytes were stained for BrdU, suggesting they were proliferating (Fig. $1 F$ ). The percentage of $\mathrm{BrdU}^{+}$astrocytes was not significantly different among astrocytes from normal or injured spinal cord (data not shown).

After coculture with the purified NSC-A in differentiation medium for $3 \mathrm{~d},>70 \%$ of OPCs differentiated into $\mathrm{O} 1^{+} \mathrm{OLs}$ 

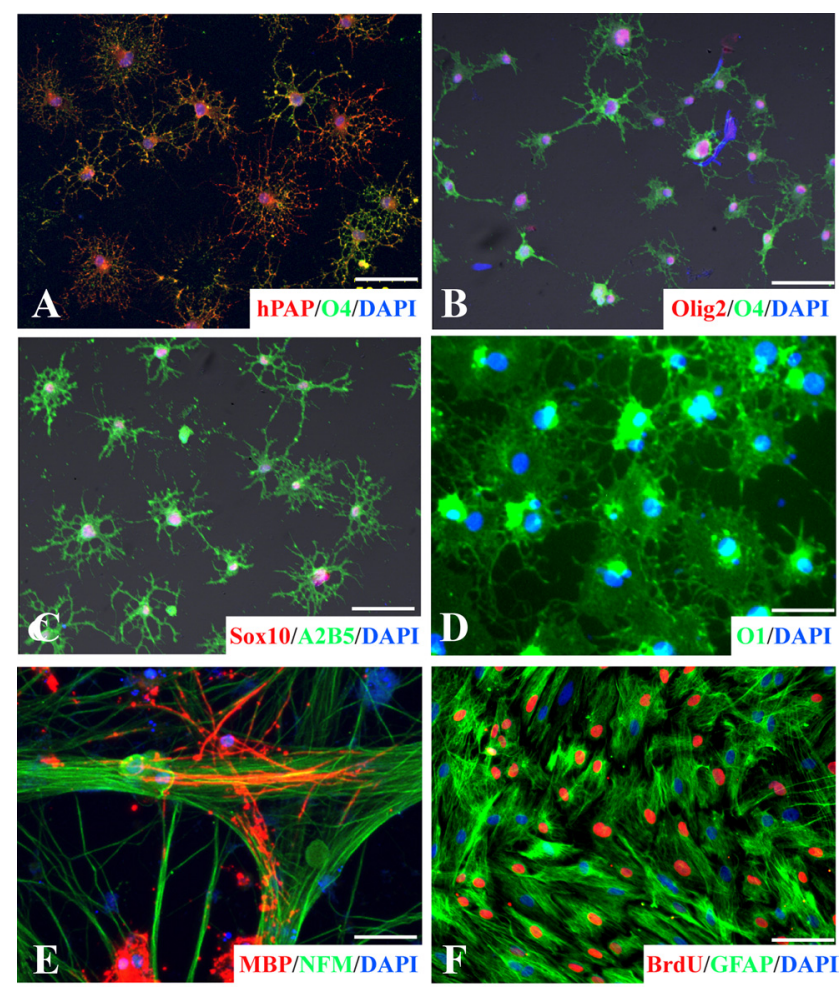

Figure 1. Characterization of OPCs and astrocytes. $A-C$, All purified OPCs express $h P A P(A)$ and OPC markers $04(\boldsymbol{A}, \boldsymbol{B})$, olig2 (B), A2B5 (C), and Sox $10(\boldsymbol{C}) \cdot \boldsymbol{D}, E, \mathrm{OPC}$ s differentiate into $01^{+} \mathrm{OLs}$ after withdrawal of FGF2 and PDGF-aa for $3 \mathrm{~d}(\boldsymbol{D})$, and $\mathrm{MBP}^{+} \mathrm{OL}$ s to form myelin around the axons after coculture with DRG neurons for 2 weeks $(\boldsymbol{E})$. $\boldsymbol{F}$, All purified astrocytes from 1 month postinjury spinal cord express GFAP. Some astrocytes are still proliferating as evidenced by BrdU incorporation. Scale bar, $50 \mu \mathrm{m}$.

(Fig. $2 A, G$ ). However, the percentage of OPCs that differentiated into $\mathrm{O}^{+}$OLs significantly decreased to $35 \%$ and $37 \%$, respectively, after cocultured with 1wISC-A and 1mISC-A (Fig. $2 C, E, G)$. In contrast, the percentage of OPCs that differentiated into GFAP-positive astrocytes significantly increased from $24 \%$ in the coculture with NSC-A to $63 \%$ and $67 \%$ in coculture with 1wISC-A or $1 \mathrm{mISC}-\mathrm{A}$, respectively (Fig. $2 B, D, F, G$ ). These results indicate that astrocytes from the injured spinal cord inhibit OL differentiation of OPCs with concurrent promotion of their astrocyte differentiation.

To further determine whether inhibition of OL differentiation by astrocytes from the injured spinal cord is mediated by soluble factors, the CMs from the purified astrocytes were obtained. After differentiation in NSC-A CMs for $3 \mathrm{~d}$, the percentage of OPCs that differentiated into $\mathrm{O}^{+}$OLs was $70 \%$, which was not significantly different from $84 \%$ in the control differentiation medium (Fig. $3 A, D)$. However, only $54 \%$ and $30 \%$ OPCs differentiated into $\mathrm{O}{ }^{+}$ OLs in CMs of 1wISC-A or 1mISC-A, respectively (Fig. 3B-D). Both were significantly lower compared with control differentiation media $(p<0.05)$. The percentage of OPCs which differentiated into $\mathrm{GFAP}^{+}$astrocytes in CMs of NSC-, 1wISC-, or 1mISC-A was $20 \%$, $36 \%$ and $62 \%$, respectively (Fig. $3 A-D$ ). All were significantly higher compared with $5 \%$ in control differentiation media $(p<0.05)$. Western blots confirmed that CMs of astrocytes from injured spinal cord significantly decreased and increased, respectively, the expression of MBP and GFAP in differentiated OPCs. To further test whether astrocytes from the injured spinal cord will inhibit OL maturation and myelination, OPCs were differentiated for $3 \mathrm{~d}$ to become $\mathrm{O}^{+}$OLs which were then added to the purified DRG neurons to
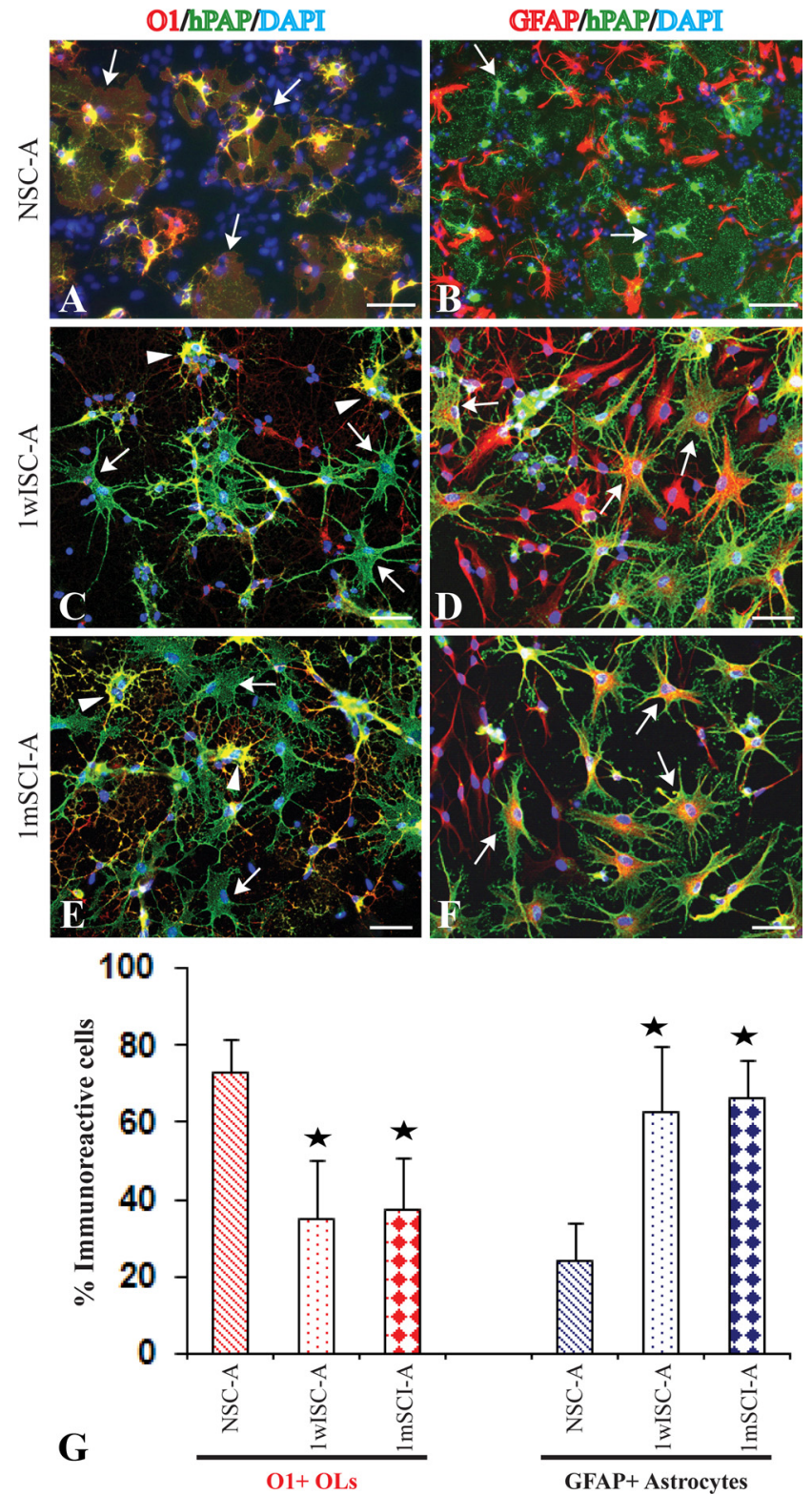

Figure 2. Active astrocytes from the injured spinal cord inhibit $0 \mathrm{~L}$ differentiation of cocultured OPCS. Three days after being cocultured with astrocytes from normal spinal cord, the majority of OPCs labeled by hPAP differentiate into $01^{+} \mathrm{OL}$ with membrane sheets, the typical morphology of mature $\mathrm{OLs}\left(\boldsymbol{A}\right.$, arrows). Most hPAP ${ }^{+} \mathrm{OPCs}$ do not differentiate into $\mathrm{GFAP}^{+}$ astrocytes ( $\boldsymbol{B}$, arrowheads). However, in the coculture with astrocytes from 1 week ( $\boldsymbol{C}$, arrows) or 1 month ( $\boldsymbol{E}$, arrows) postinjury spinal cord, most OPCs labeled by hPAP fail to differentiate into $01^{+} \mathrm{OLs}$. OPCs, which differentiate into $01^{+} \mathrm{OLs}$, lack the complex membrane sheets $(\boldsymbol{C}$, $\boldsymbol{E}$, arrowheads). Most OPCs labeled by hPAP (green) differentiate into $\mathrm{GFAP}^{+}$astrocytes (red) ( $\boldsymbol{D}, \boldsymbol{F}$, arrows). Quantitative data confirm that reactive astrocytes from the injured spinal cord significantly decrease $\mathrm{OL}$ differentiation with a concurrent increase of astrocyte differentiation of cocultured OPCS $(G)$. Data represent the mean \pm SD from four repeated experiments from separately generated cultures; ${ }^{*} p<0.05$. Scale bar, $50 \mu \mathrm{m}$.

coculture for 12 more days. In control media or NSC-A CM (Fig. $3 F$ ), OPCs matured into MBP OLs which formed myelin along the axons. In 1wISC-CM (Fig. 3G) or 1mISC-A CM (Fig. 3H), OPCs differentiated into mature $\mathrm{MBP}^{+} \mathrm{OLs}$. However, they failed to myelinate the axons they contacted. These results showed that soluble factors in CMs of astrocytes from the injured spinal cord inhibit OL differentiation and myelination of OPCs.

BMP signaling is well known for its roles to induce astrocyte differentiation and inhibit OL differentiation of OPCs. We used 
Western blot analysis to examine the expression of BMPs in astrocytes. Expression of BMP2, but not BMP4, was detected in NSC-A. However, expression of both BMP2 and BMP4 was significantly increased in 1wISC-A or 1mISC-A (Fig. $4 A$; supplemental Fig. $2 A$, available at www.jneurosci.org as supplemental material). The expression of pSMAD $1 / 5 / 8$, the downstream target transcription factors of BMP receptors, was also significantly increased in astrocytes from the injured spinal cord. We further use ELISA to directly detect the secretion of BMPs in astrocyte CMs. Consistent with the Western blot results, BMP2 was detected in all three astrocyte CMs. But its concentration was dramatically higher in CMs of 1wISCor 1mISC-A compared with NSC-A (Fig. $4 B)$. Since an ELISA kit is not available for rat BMP4, we were unable to detect its expression in CMs. To test whether astrocyte CMs activated BMP pathway in OPCs, we examined its expression of phosphorylated SMAD 1/5/8, the downstream target of BMP receptors. As shown in Figure $4 C$, expression of pSMAD in OPCs was significantly increased after treating with CMs of 1wISC- or 1mISC-A. The activation of pSMAD is concurrent with the increasing expression of GFAP and the decreasing expression of $\mathrm{MBP}$ and PLP. To further determine whether BMP signaling mediated the effects of astrocyte CMs in OPC differentiation, we blocked its activity by using BMP receptor-specific antagonist, noggin (Fig. 4C). Addition of noggin $(200 \mathrm{ng} / \mathrm{ml})$ to the CMs of 1 wISC or $1 \mathrm{mISC}$-A partially reversed the decrease of MBP and PLP and increase of GFAP in the differentiated OPCs, respectively (Fig. 4C). Our immunohistochemistry results also showed that addition of noggin significantly decreased the percentage of OPCs differentiating into $\mathrm{GFAP}^{+}$astrocytes from $30 \%$ to $14 \%$ in 1wISC-A CMs and $50 \%$ to $32 \%$ in $1 \mathrm{mISC}-\mathrm{A}$ CMs (Fig. $4 D-G$ ). Meanwhile, noggin treatment significantly increased the percentage of OPCs differentiating into $\mathrm{O}^{+}$OLs from $54 \%$ to $68 \%$ in 1 wISC-A CMs and from $30 \%$ to $54 \%$ in $1 \mathrm{mISC}-\mathrm{A}$ CMs (Fig. $4 D-G$ ). Noggin treatments did not change either the percentage of $\mathrm{O}^{+}{ }^{+} \mathrm{OLs}$ or GFAP ${ }^{+}$ astrocytes in control medium or NSA-A CMs. These results showed that increasing expression of BMPs is an important mechanism by which astrocytes from 1 week or 1 month ISC inhibit OL differentiation of OPCs with a concurrent promotion of astrocyte differentiation.

Previous studies showed that Notch (Wang et al., 1998) and LIF (Fukuda et al., 2004) signaling are also inhibitory for OL differentiation. We examined the expression of Notch ligand, Jagged 1, and LIF in the astrocytes by Western blot. Expression of Jagged1 was detected, but not significantly different among NSC-, 1wISC-, or 1mISC-As (data not shown). Expression of LIF was not detected in either NSC-, 1wISC-, or 1mISC-A. To test whether expression of BMP was limited in astrocytes around the injured area, we purified astrocytes from the cervical, the injured thoracic, and lumbar spinal cord at 1 week postinjury. The ex-
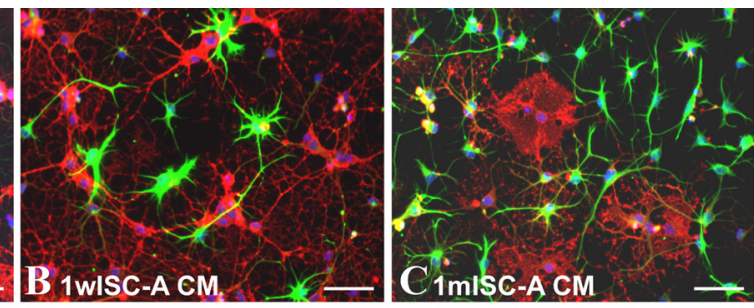

Figure 3. Conditioned media from reactive astrocytes from the injured spinal cord inhibit OL differentiation of cocultured OPCS. OPCs are differentiated for $3 \mathrm{~d}$ in control basal medium, NSC-A, $1 \mathrm{wISC}-\mathrm{A}$, or $1 \mathrm{mISC}-\mathrm{A}$. In basal medium or NSC-A CM, the mith few into GFAP astrocytes $(A$, green). However, in CM of ISC $1 W-A(B, D)$ rons for $12 \mathrm{~d}$ in CM from NSC-A, OPCS differentiate into $\mathrm{MBP}^{+}$mature $\mathrm{OLS}$, which form myelin along axons. However, myelin formation is not observed, although OPCs differentiate into MBP ${ }^{+}$OLs in CM of 1wISC-A (G) or 1mISC-A (H). Data in D represent the mean \pm SD from four independent experiments; ${ }^{*} p<0.05$. Scale bar, $50 \mu \mathrm{m}$.

pression of BMP4 was high in the astrocytes from the injured thoracic spinal cord. However, its expression is low in lumbar astrocytes and not detectable in cervical astrocytes (supplemental Fig. $2 B$, available at www.jneurosci.org as supplemental material). The low expression of BMP4 in lumbar astrocytes was probably due to the fact that they were located closely to the injury site. The expression of BMP4 was also directly examined in the injured spinal cord. Expression of BMP4 was not detected in the astrocytes in the normal spinal cord. But its expression was dramatically increased in astrocytes in the injured spinal cord at 1 week or 1 month postinjury (supplemental Fig. $3 A-C$, available at www.jneurosci.org as supplemental material). These results showed that up-regulation of BMP4 in astrocytes was specific to the contused area. To directly test whether injury to cultured astrocytes could increase the expression of BMP4 in vitro, the purified 1wISC-A was injured by scratching. Two days after the scratching injury, GFAP expression was dramatically increased in the astrocytes located in the scratching edge (supplemental Fig. $4 A$, available at www.jneurosci.org as supplemental material). The expression of BMP4 was also significantly increased (supplemental Fig. $4 B$, available at www.jneurosci.org as supplemental material).

\section{Discussion}

Progressive gliosis appears in demyelinated areas in MS lesions or SCI. In a chronically demyelinated injury, the bare axons are 

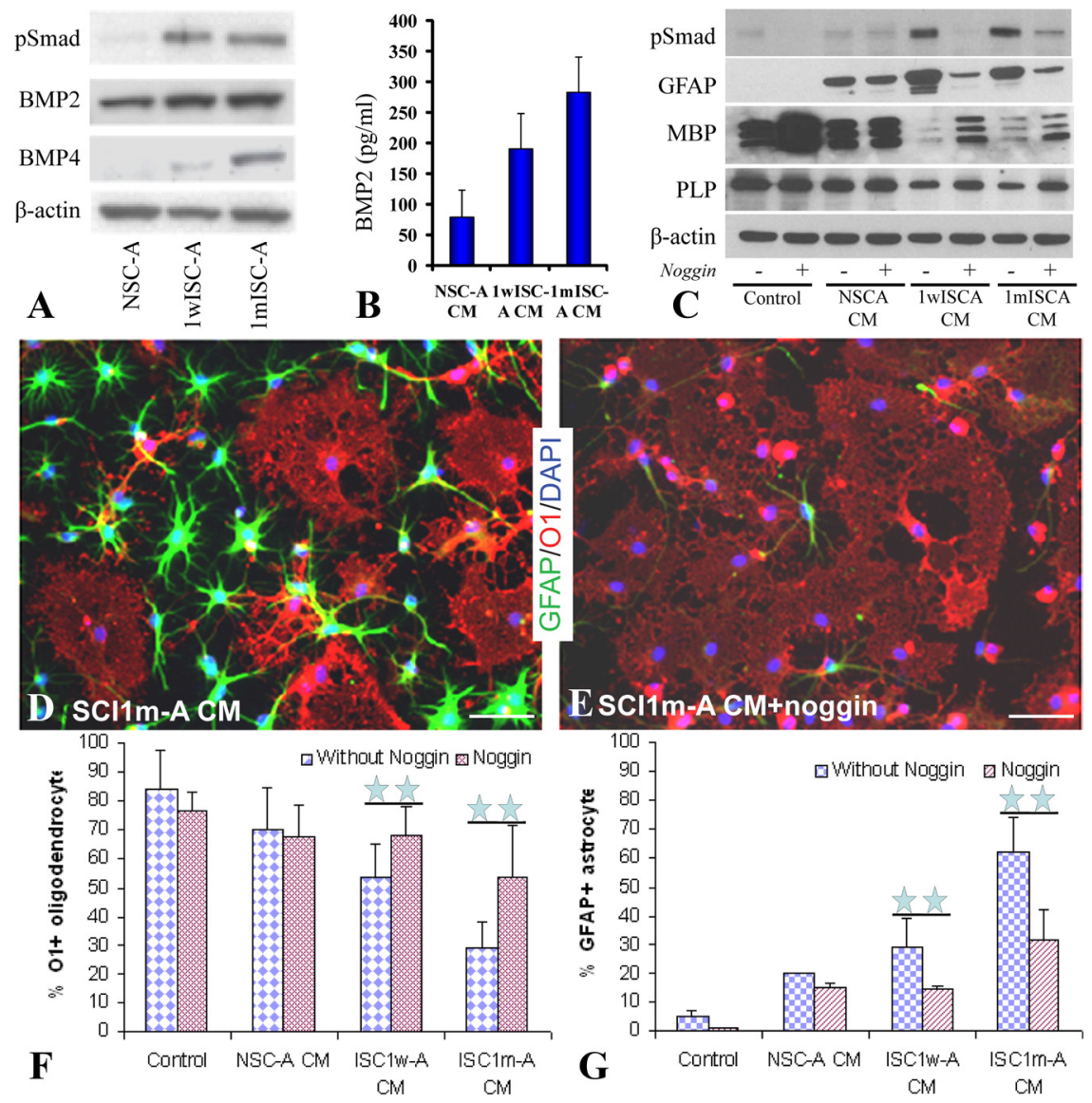

Figure 4. ISC1w-A and ISC1m-A inhibit OL differentiation of OPCs by increasing expression of BMPs. $\boldsymbol{A}, \boldsymbol{B}$, Expression of $B M P 2$ and 4 is significantly increased in ISC1w- or ISC1m-A (A) or their CMs (B). C, CMs of 1wISC- or 1mISC-A increase the expression of pSMAD 1/5/8 in OPCs with decreasing expression of MBP and increasing expression of GFAP. Addition of noggin blocks the expression $\mathrm{PSMAD}$ and significantly increases and decreases the expression of MBP and GFAP, respectively (C). D-G, Immunohistochemistry further confirmed that blocking BMP signaling by noggin reverse the inhibition of the ISC1W or ISC1m-A CMs to significantly increase the number of $\mathrm{OPCS}$ differentiated into $\mathrm{OLS}$ with a concurrent decrease of astrocyte differentiation. $\mathrm{OL}$ or astrocyte differentiation in control basal medium or NSC-ACMs, however, was not changed by the addition of noggin. Data in $\boldsymbol{F}$ and $G$ represent the mean \pm SD from four repeated experiments; ${ }^{* *} p<0.01$. Scale bar, $50 \mu \mathrm{m}$.

often surrounded by processes of reactive astrocytes. The role of astrogliosis in demyelination/remyelination remains unknown and is controversial (Williams et al., 2007). A previous study showed that transplantation of astrocytes which had been cultured for a long time significantly decrease OL remyelination of co-grafted OPCs in the demyelinated spinal cord (Blakemore et al., 2003). The reactive astrocytes in the chronic demyelinated CNS of taiep rats may contribute to the remyelination failure by endogenous or grafted OPCs (Foote and Blakemore, 2005). However, the effects of astrocytes from the injured CNS on OL differentiation have not been directly tested. In this study, we provided direct evidence that reactive astrocytes from the injured spinal cord or their CMs inhibited OL differentiation of OPCs and promoted their astrocyte differentiation. These results suggest that astrocytes in the injured CNS may decrease remyelination by inhibiting OL differentiation and maturation of OPCs. Nevertheless, our results also showed that only astrocytes from injured spinal cord, but not from normal spinal cord, inhibit OL differentiation and myelination. In fact, astrocytes play important roles in myelination during development. For example, astrocytes from postnatal brain promote myelination of cultured OLs by releasing LIF in response to ATP liberated from cocultured DRGN axons firing action potentials (Ishibashi et al., 2006).
The dysfunction of astrocytes caused by GFAP mutation leads to abnormal myelination, including reduced myelin thickness in the spinal cord (Liedtke et al., 1996). Therefore, the effects of astrocytes on myelination and/or remyelination may be dependent on its "activation" status. Astrocytes undergo dramatic changes following CNS injury, which may transform the astrocytes from facilitation to inhibition of myelination.

Understanding mechanisms by which reactive astrocytes inhibit OL remyelination may lead to new therapeutic targets to enhance remyelination and functional recovery after many neurological diseases including MS and SCI. Our results showed that expression of BMPs was significantly increased in astrocytes or their CMs from the injured spinal cord compared with that from NSC. Importantly, blocking BMP pathway with noggin reversed the inhibition of OL differentiation by CM of astrocytes from the injured spinal cord. These results indicate that BMP signaling is one of the major inhibitors from astrocytes of injured spinal cord for OL differentiation. Previous studies showed that BMP signaling enhances astrocyte differentiation and inhibits the oligodendrocyte differentiation from cultured neural stem cells or OPCs in vitro (Mabie et al., 1997; See et al., 2004; Cheng et al., 2007). In vivo, the presence of inhibitory BMP signaling in the dorsal neural tube represses the development of OLs from this area (Wada et al., 2000; Hall and Miller, 2004). Normal oligodendrogenesis in the ventral spinal cord is also inhibited when BMP signaling is induced (Mekki-Dauriac et al., 2002; Hall and Miller, 2004). Nevertheless, our results showed noggin treatment only partially rescued OL differentiation of OPCs although it blocked almost all BMP activity as shown by expression of pSMAD $1 / 5 / 8$. These results indicate that other factors, such as jagged 1 (John et al., 2002) or hyaluronan (Back et al., 2005), may also play important role in the inhibition of OL differentiation by astrocytes from the injured spinal cord.

Although endogenous OPCs are present in the demyelinated areas and some even closely contact the affected axons, they fail to differentiate and mature into myelinating OLs to remyelinate the demyelinated axons in MS lesions or SCI (Chang et al., 2002; Wolswijk, 2002; Wilson et al., 2006). Inhibitory signals in the demyelinated lesions may prevent OPCs from differentiation and maturation. Upregulated BMP signaling in reactive astrocytes may represent an important factor to inhibit OL remyelination in the injured CNS. Expression of BMPs dramatically increases after traumatic SCI (Setoguchi et al., 2004), local chemical demyelination (Fuller et al., 2007) or EAE (Ara et al., 2008). Moreover, the neuronal and oligodendrocyte differentiation from grafted neural stem cells in the injured spinal cord are increased by blocking BMP signaling with expression of noggin (Setoguchi et al., 2004). Together, these studies suggest that manipulation of BMP signaling may provide a new therapeutic strategy to enhance remyeli- 
nation from endogenous or/and grafted OPCs after multiple sclerosis or SCI.

\section{References}

Anderson JM, Hampton DW, Patani R, Pryce G, Crowther RA, Reynolds R, Franklin RJ, Giovannoni G, Compston DA, Baker D, Spillantini MG, Chandran S (2008) Abnormally phosphorylated tau is associated with neuronal and axonal loss in experimental autoimmune encephalomyelitis and multiple sclerosis. Brain 131:1736-1748.

Ara J, See J, Mamontov P, Hahn A, Bannerman P, Pleasure D, Grinspan JB (2008) Bone morphogenetic proteins 4, 6, and 7 are up-regulated in mouse spinal cord during experimental autoimmune encephalomyelitis. J Neurosci Res 86:125-135.

Back SA, Tuohy TM, Chen H, Wallingford N, Craig A, Struve J, Luo NL, Banine F, Liu Y, Chang A, Trapp BD, Bebo BF Jr, Rao MS, Sherman LS (2005) Hyaluronan accumulates in demyelinated lesions and inhibits oligodendrocyte progenitor maturation. Nat Med 11:966-972.

Blakemore WF, Gilson JM, Crang AJ (2003) The presence of astrocytes in areas of demyelination influences remyelination following transplantation of oligodendrocyte progenitors. Exp Neurol 184:955-963.

Chang A, Tourtellotte WW, Rudick R, Trapp BD (2002) Premyelinating oligodendrocytes in chronic lesions of multiple sclerosis. N Engl J Med 346:165-173.

Cheng X, Wang Y, He Q, Qiu M, Whittemore SR, Cao Q (2007) Bone morphogenetic protein signaling and olig1/2 interact to regulate the differentiation and maturation of adult oligodendrocyte precursor cells. Stem Cells 25:3204-3214.

Foote AK, Blakemore WF (2005) Inflammation stimulates remyelination in areas of chronic demyelination. Brain 128:528-539.

Franklin RJ, ffrench-Constant C (2008) Remyelination in the CNS: from biology to therapy. Nat Rev Neurosci 9:839-855.

Fukuda S, Kondo T, Takebayashi H, Taga T (2004) Negative regulatory effect of an oligodendrocytic bHLH factor OLIG2 on the astrocytic differentiation pathway. Cell Death Differ 11:196-202.

Fuller ML, DeChant AK, Rothstein B, Caprariello A, Wang R, Hall AK, Miller RH (2007) Bone morphogenetic proteins promote gliosis in demyelinating spinal cord lesions. Ann Neurol 62:288-300.

Hall AK, Miller RH (2004) Emerging roles for bone morphogenetic proteins in central nervous system glial biology. J Neurosci Res 76:1-8.

Ishibashi T, Dakin KA, Stevens B, Lee PR, Kozlov SV, Stewart CL, Fields RD (2006) Astrocytes promote myelination in response to electrical impulses. Neuron 49:823-832.

John GR, Shankar SL, Shafit-Zagardo B, Massimi A, Lee SC, Raine CS, Brosnan CF (2002) Multiple sclerosis: re-expression of a developmental pathway that restricts oligodendrocyte maturation. Nat Med 8:1115-1121.

Keirstead HS, Nistor G, Bernal G, Totoiu M, Cloutier F, Sharp K, Steward O
(2005) Human embryonic stem cell-derived oligodendrocyte progenitor cell transplants remyelinate and restore locomotion after spinal cord injury. J Neurosci 25:4694-4705.

Kisseberth WC, Brettingen NT, Lohse JK, Sandgren EP (1999) Ubiquitous expression of marker transgenes in mice and rats. Dev Biol 214:128-138.

Liedtke W, Edelmann W, Bieri PL, Chiu FC, Cowan NJ, Kucherlapati R, Raine CS (1996) GFAP is necessary for the integrity of CNS white matter architecture and long-term maintenance of myelination. Neuron 17:607-615.

Mabie PC, Mehler MF, Marmur R, Papavasiliou A, Song Q, Kessler JA (1997) Bone morphogenetic proteins induce astroglial differentiation of oligodendroglial-astroglial progenitor cells. J Neurosci 17:4112-4120.

Mekki-Dauriac S, Agius E, Kan P, Cochard P (2002) Bone morphogenetic proteins negatively control oligodendrocyte precursor specification in the chick spinal cord. Development 129:5117-5130.

Raine CS (2008) Multiple sclerosis: classification revisited reveals homogeneity and recapitulation. Ann Neurol 63:1-3.

See J, Zhang X, Eraydin N, Mun SB, Mamontov P, Golden JA, Grinspan JB (2004) Oligodendrocyte maturation is inhibited by bone morphogenetic protein. Mol Cell Neurosci 26:481-492.

Setoguchi T, Nakashima K, Takizawa T, Yanagisawa M, Ochiai W, Okabe M, Yone K, Komiya S, Taga T (2004) Treatment of spinal cord injury by transplantation of fetal neural precursor cells engineered to express BMP inhibitor. Exp Neurol 189:33-44.

Skripuletz T, Bussmann JH, Gudi V, Koutsoudaki PN, Pul R, MoharreghKhiabani D, Lindner M, Stangel M (2010) Cerebellar cortical demyelination in the murine cuprizone model. Brain Pathol 20:301-312.

Talbott JF, Cao Q, Enzmann GU, Benton RL, Achim V, Cheng XX, Mills MD, Rao MS, Whittemore SR (2006) Schwann cell-like differentiation by adult oligodendrocyte precursor cells following engraftment into the demyelinated spinal cord is BMP-dependent. Glia 54:147-159.

Trapp BD, Nave KA (2008) Multiple sclerosis: an immune or neurodegenerative disorder? Annu Rev Neurosci 31:247-269.

Wada T, Kagawa T, Ivanova A, Zalc B, Shirasaki R, Murakami F, Iemura S, Ueno N, Ikenaka K (2000) Dorsal spinal cord inhibits oligodendrocyte development. Dev Biol 227:42-55.

Wang S, Sdrulla AD, diSibio G, Bush G, Nofziger D, Hicks C, Weinmaster G, Barres BA (1998) Notch receptor activation inhibits oligodendrocyte differentiation. Neuron 21:63-75.

Williams A, Piaton G, Lubetzki C (2007) Astrocytes: friends or foes in multiple sclerosis? Glia 55:1300-1312.

Wilson HC, Scolding NJ, Raine CS (2006) Co-expression of PDGF alpha receptor and NG2 by oligodendrocyte precursors in human CNS and multiple sclerosis lesions. J Neuroimmunol 176:162-173.

Wolswijk G (2002) Oligodendrocyte precursor cells in the demyelinated multiple sclerosis spinal cord. Brain 125:338-349. 